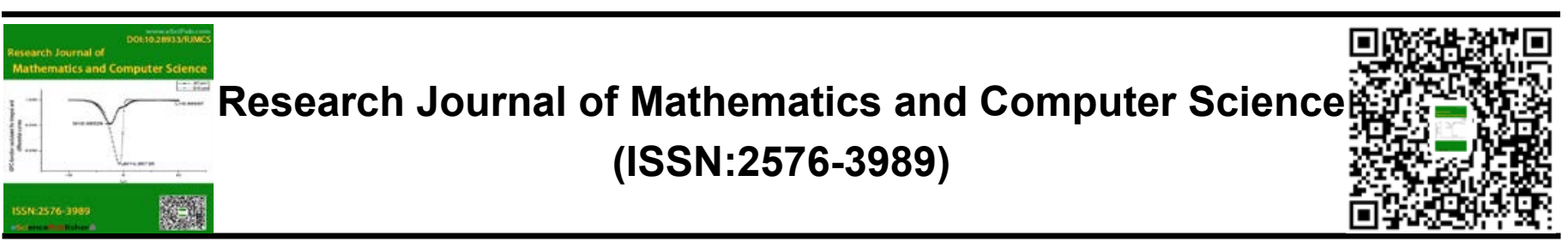

\title{
Traffic Shaping for Congestion Control
}

\author{
Kuyoro Shade O., Okolie Samuel O. and Oyebode Aduragbemi*
}

Department of Computer Science, Babcock University, Nigeria

\begin{abstract}
The problem of congestion is not new to the field of telecommunications; its root can be traced back to the very foundations of the internet. Most, if not all networks, are currently faced with this problem. The distribution of resources on the network is very vital. The fact that resources are limited brought about major problem such that these limited resources would be shared among various workstations and nodes. As the network grows, and the number of workstations and nodes increases; resources become integral and if not properly managed no meaningful work will be done in such networks. Congestion control, which involved managing resources when the network utilization becomes so high, is of utmost importance so as to avoid congestion collapse. *Correspondence to Author: Oyebode Aduragbemi Department of Computer Science, Babcock University, Nigeria The complex calculations and computation carried out in the How to cite this article:

Kuyoro Shade O., Okolie Samuel O. and Oyebode Aduragbemi. Traffic existing solutions, increase utilization as well as the problem of congestion. Traffic shaping algorithms provide solutions with little overhead and thus would be preferred to use in medium sized networks. This work proposes traffic shaping as a better solution for congestion control especially in medium size networks.

\section{eSciencePublisher 2} eSciPub LLC, Houston, TX USA. Website: http://escipub.com/

Keywords: Congestion; Algorithms; Traffic Shaping; Overhead; Networking
\end{abstract}




\section{INTRODUCTION}

Today, it can be said that the world is a global village, the internet which was created far back as January 1983, has revolutionized communication, business and also wealth creation (Vidyasagar \& VidyaShankar, 2013). The internet and basically all kinds of networks can be viewed logically as a queue of packets. Packets, which are unit of data fragments are constantly being transmitted between nodes, this forms the basic foundation of networking.

Networks perform the tasks of packet transmission between nodes thereby reducing the number of packets remaining of the queue waiting to be transmitted. As network grows in terms of usage, certain issues begin to emerge. As the utilization of a particular network increases so also does the number of nodes added to the queue. As this continues, a bottleneck situation will arise such that the network cannot process and transmit these packets fast enough, thereby allowing the packets to be continually added to the queue which will have to wait a long while to be transmitted leading to network congestion. This degraded the turnaround time of the network causing packets to be timed out, and then retransmitted back to the node that has a queue filled with unprocessed packets.

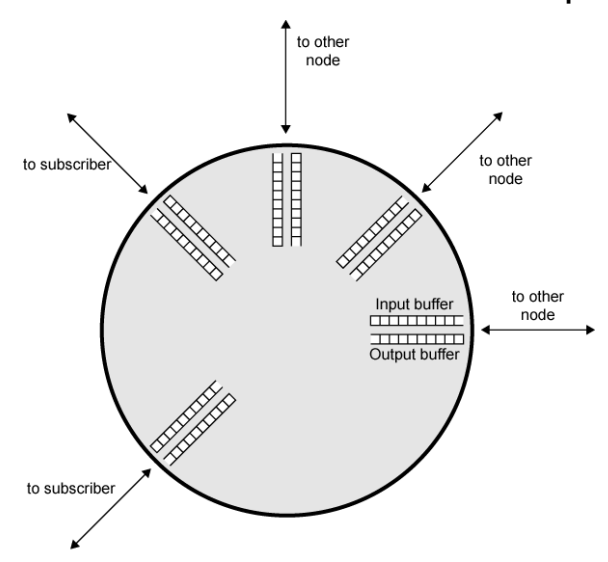

Figure 1: The logical description of a node. (Spring, 2003)

Figure 1 shows the model of a node and nodes behaviour. The input and output buffer store packets sent and also received from other nodes waiting to be transmitted. A network comprised of various nodes and all the nodes account for packets queued up and waiting to be transmitted.

Congested nodes often gather up so much packets or they can also gather very little but are unable to either send or receive. The internet itself has also suffered many congestion issues, 1986 saw one of the first congestion issues related to the internet, these led to a drastic reduction in throughput and productivity of the network as a whole (Miller \& Hsiao, 2014); hence proper understanding of network congestion is of paramount importance. Congestion occurs when the demand for a particular piece of packet or resource is greater than what is available to be sent at that point in time. (Jain, 1990) Most network administrators have misguided opinions about this and also how to solve the problem. Some of the misguided views include:

1. Congestion is caused by lack of sufficient buffer memory, so the solution to this is that memory has to become cheap enough thereby allowing for infinite storage.

2. Slow links between connecting nodes is the cause of congestion, thus, having high speed connecting links will solve the problem.

3. Slow processing power will lead to congestion; hence, having high speed processors will solve the problem.

All these are some of the assumptions made by network administrators which often lead to improper network planning and implementation. 
On the contrary, proper network protocol design and implementation is needed to establish a network to combat congestion. Any of the abovementioned, would reduce the overall throughput and also reduce performance but will add more congestion to the network.

Infinite buffer storage, though not a bad idea, cannot solve the problem of congestion. Even though, small memory buffers when faced with too much traffic, suffer from buffer overflow and loss of packets; however, having infinite buffer storage during heavy traffic would result in long queues of packets, delays and so on. Finally, by the time packets leave the queue, so much time would have passed and these packets would be times out.

Also, having high speed links between nodes will not make much difference to solve the problem of congestion, at the time when the internet began, the speed for nodes and workstation available as at the time was not more than 300 bits per seconds $(300 \mathrm{~b} / \mathrm{s})$. Gradually with increase in technology, speeds of up to 1.5 megabits per seconds $(1.5 \mathrm{Mb} / \mathrm{s})$ was attainable with the introduction of Local Area Networks (LANs) and also Ethernet speeds of about 10 megabits per seconds $(10 \mathrm{Mb} / \mathrm{s})$ was possible (Jain, 1990). It was at this point in history that the issue of congestion started to gain recognition due to a mismatch such that fast high speed LANs were connected via slow links and slow nodes that cannot keep up to the speed of these links. Today, when it comes to the speed of data or internet connections, it can be up to Gigabits or even Terabits but the issue of congestion still persists. Therefore having high speed links is obviously not the solution. Also, the same situation describes the slow processors, having high speed processors which can lead to a mismatch.

Congestion is a difficult and challenging problem to solve, and the resources are limited. Resources such as router processing time, data, link throughput and so on, are limited and can be made available to specific number of nodes for limited amount of time. Also, several networks are designed and implemented with several policies which inform the methods or algorithms that can be employed to control congestion on a particular network. Thus, implying that a standard algorithm cannot be employed. Also, requirements and specifications for congestion control methods and algorithms differ from network to network meaning that a method of congestion control on one network may not necessarily be efficient on another network.

Congestion is a persistent problem in which no one specific solution can eradicate. Thus, there is need to find an efficient managing the problem. Algorithms, schemes, methods have been deployed to effectively contain congestion (Jain, 1990). Congestion Control entails adequately maintaining the efficiency of a network when it is operating on a high load. This is an issue which operates at network layer, it refers to how the network responds when there is too much data residing in the network that can be sent considering packet delays and also packets loss. Congestion control is integral to network performance; it acts as a means of ensuring stable and adequate control over data traffic in the network.

Researchers tends to assume that congestion control is the same as flow control; however, in application these are two different concepts (Rysavy Research, 2014). Flow control refers to mechanisms set in place to control the flow and movement of data packets between the sending and receiving nodes while congestion control deals with traffic control placed into the network layer by the transport layer. The receiving node is what is concerned with when talking of flow control; it ensures that the sending node is only able to send what can be handled by the receiving node. This is paramount so as not to overwhelm and also overload the node with so much data packets such that it will eventually breaks down. Flow control and congestion control are both traffic control mechanisms and should not be confused for one another.

Congestion control can be viewed as a way of ensuring that every workstation or node connect 
across the network, is guaranteed of a fair level or amount of access to network resources at any given instance and time. Congestive collapse is a scenario in which little or no significant and meaningful work is done on a network due to the effects of congestion. At this point the system utilization is so high and throughput so low, that no significant job is done on the network. This can be referred to as a bottleneck situation. Congestion need to be efficiently managed as well as controlled (Rysavy Research, 2014).

This work presents congestion control methods and algorithms; respective improvements on congestion handling; existing gap as well as propose traffic shaping method as an efficient way of handling congestion. Congestion algorithms refers to the ways and techniques networks administrators employ to manage congestion in their networks. In this age of Quality of Service, modern networks have the capacity to support traffic beyond the well-known or traditional data types such emails, file sharing etc. But today, data networks are increasingly being used as sharing medium for a much more delicate and time bound forms of data such as voice and data. (Aaron Balchunas, 2010) Congestion control now becomes a much more sensitive issue due to the sensitive nature of data being handled by networks.

\section{Literature Review}

Over the years many algorithms, strategies coupled with procedures and methods have been employed and adopted to control and manage congestion.

In 2016, Hu and Guo, Chinese scientists developed a "robust control model" to tackle congestion issues in wireless networks. The work, which was on congestion control in wireless software, defined networks with propagation delay and external interference using a robust control approach. It was implemented through the use of a centralized controller embedded into a network simulation. This controller was tasked with the work of retrieving information from all nodes and devices on the network. This is very crucial due to the fact that the congestion control algorithm will used the information supplied as its parameters. A nonpreemptive scheme was used in the control system. The congestion problem is difficult as it is but considering a network environment where scheduling is also of importance, a nonpreemptive scheme is adopted which offers stability to the network parameters gotten from various nodes and devices to maximize the overall throughput of the system. The solution provided by $\mathrm{Hu}$ and Guo appears very complex, requiring complex computations using mathematical theorems which might not be easily understood by the network administrators. Jain 1990 opined that schemes and algorithms adopted to tackle the congestion problem must have a low overhead. This property is very vital and must be present in any algorithm to be adopted. It should not increase traffic on the network or reduce the overall throughput of the networks. The solution provided by $\mathrm{Hu}$ and Guo, 2016; due to the computational complexity, would greatly increase the workload on the system and thus the overall traffic therefore this solution may not be preferred in certain kinds of networks.

Mohamed et al 2016, carried out a study on understanding the effects of congestion on network performance. The study begins by introducing the concept of congestion. In the world of research, no piece of literature clearly gives the exact detail on the types of congestion. The study also goes on and gives more details has to why network congestion can be termed unavoidable. The access link can be said to be the primary bottleneck source, due to the fact that the network access link is the door to the network, this door is actually a network resource and its limited in size and also scope, so upon entry into the network congestion has already taken place. Therefore, congestion is a necessary evil every network must face; however how this problem is managed is what is of paramount importance. Monitoring traffic conditions directly is the most logical precaution that can taken to reduce the risk of congestion to 
the minimal probability. When dealing with huge networks, congestion detection has to be a proactive process, with certain strategies and monitoring policies being implemented, it is possible have a good foresight to when congestion will likely occur in a network. Observing the utilization and the throughput of the network, it is possible to tell a network work will likely be congested. The study did not propose a solution to the problem of congestion, it only presents review of various techniques and respective classifications. It however, offers work an insight into the problem of congestion.

Various methods of congestion control have been adopted, and most of which offer dynamic solutions and procedures. One of such is the application of heuristics to the problem of congestion. Heuristics (trial and error technique) can be described as a technique of solving problem, which makes use of learning, understanding and discovery, though it does not guarantee a perfect result or outcome, but it provides a means of satisfying the immediate objectives.

Jiao et al 2014 presents a study on the application of heuristics to the problem of congestion in networks. A fast heuristics algorithm and two mathematical theorems were employed to minimize congestion in the networks. Data packets were transmitted via routes, the routes can be seen as paths from source to destination. If these routes can be known, how busy the route is can be determined and if its busy packets can be transferred through other routes. The most feasible path for packets to travel is calculated and also other techniques such as round-robin in which token is given to nodes was also employed. Although, these had a sufficiently high overhead, it would prove very useful in real-time systems. The fact that the algorithm has a high overhead, limits the whole application of the algorithms and cannot be employed by all networks.

Shah et al 2015, reviewed congestion control algorithms and respective implementation focusing on wireless sensor networks.
Considering the delicate nature and also functionality of sensor networks, which operate and function in real-time, congestion control is of vital importance to sensor networks in which a down time of even seconds can be critical to network performance. Taking into considerations all the various methods that have previously been used in sensor networks to combat congestion such as routing protocols coupled with features of congestion detection and also protocols dedicated to congestions control, were reviewed. Sensor networks consists of various individual devices all of which collect information and data from the external environment.

Shar, Nadir, \& Khan 2016 identified a sink node as a solution to congestion. A sink node is the node with which all information gathered by the various network elements are relayed to. This transfer is very crucial to the network, the information should be pass to the sink node with as little congestion as possible, which calls for an adequate congestion control mechanism. Although adequate reviews on existing techniques was done, most of these techniques will have a high overhead which will add sufficient congestion to the system.

Congestion also has span across various wireless technologies. One of the emerging technologies faced with this issue is the Mobile ad-ho Network (MANET). Technological advancement in the field of telecommunication has led to the creation of highly dynamic networks, one of such is Mobile Ad-Hoc Networks which can be described as an autonomous collection of devices (mobile devices) that offers dynamic topologies, no central administration, dynamic and ever mobile nodes and so on, information becomes easy to disseminate. (Raja \& Baboo, 2014)

Ramratan Ahirwal et al 2012 did a work on a congestion control mechanism in MANETs using bandwidth estimation. Bandwidth refers to the amount of data that can be sent per unit of time. Transmission Control Protocol (TCP) was employed. TCP uses a network congestion 
avoidance algorithm which is part of the protocol itself making it one of the widely-used protocol in telecommunications. NS-2.32 simulator was used to simulate a network of about 10 nodes without a central administrator, in infrastructure less environment of MANETs. Packets of size 512 bytes was used. The result showed that TCP performed incredibly well when it comes to congestion control and management. Although, the study recorded low overhead, it will be difficult to assume the solutions can be reduplicated in a real-life application of MANETs, since the work was simulated.

\section{Traffic Shaping}

Traffic shaping, which can also be referred to as Bandwidth Shaping or Data Packet Shaping is a method or means of controlling network traffic by setting priorities to network resources and also offering certain assurances on the bandwidth according to some predetermined policies drawn out (IPLOCATION, 2017). Traffic shaping is a traffic management technique used on computer networks, which deals with ensuring the flow control of packets moving for node to node ensuring that nodes are not overwhelmed with packets. Datagrams or packets may experience delays or a total halt in order to bring the data fragments to adhere to the traffic profile of the network.

Another method of achieving congestion control is to "shape" the traffic i.e. flow of packets before it enters into the network. This can be described as a very efficient way of managing congestion, which result in a very low overhead. The

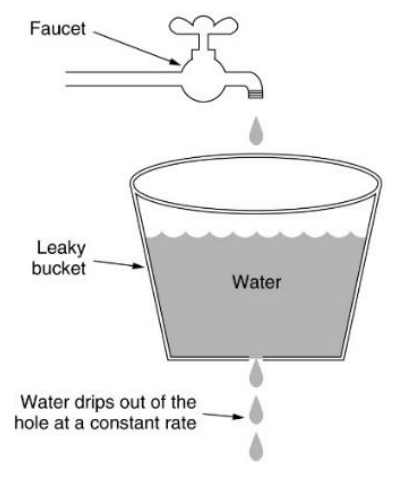

(a) algorithm does not add more congestion to the network. (Hussain, Jamwal, \& Zaman, 2015)

When the sending node is about to send data packets, at the point where connection occurs the sending node together with the carrier links agree as to a specified network traffic pattern. The data is then passed to flow strictly through the specified traffic policy. This ensures that the network is not flooded with packets and also packets are not kept too long on queues. This method of congestion control is highly efficient and very easy to adopt. (Vidyasagar et al, 2013) The Traffic shaping can be implemented with two main algorithms:

1. Leaky Bucket

2. Token bucket

Leaky Bucket: This algorithm is used to control data and traffic rates in the network. A host node or workstation can put in one packet per clock tick into a network. The algorithm is implanted as a single-server queue. The queue has a constant time (i.e. service time) to service the packets.

The buffer, abstracted as the bucket, can overflow, just the same way a bucket of water can overflow and the water spilled is overlooked and discarded. Similarly, once there is an overflow, the packets that spill over are discarded. The leaky bucket algorithms adopt a constant output rate for data packets regardless of the number of packets or bursts into the network. This leads to uniform distribution of packets through the network.

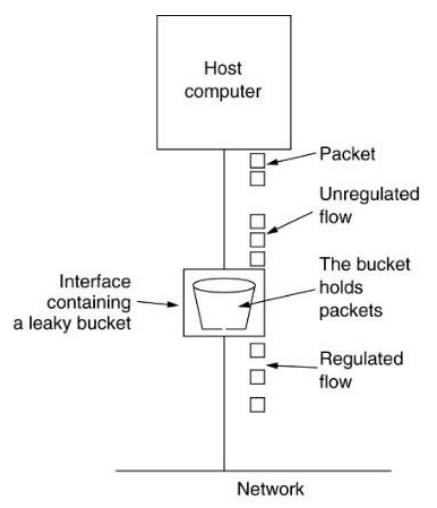

(b)

Figure 2: A representation of the leaky bucket algorithm (Vidyasagar et al, 2013) 
Token Bucket: The token bucket algorithm in contrast allows the output rate for data packets to vary. The rate at which packets are passed to the network depends on the size of the packets (i.e. the rate or size of bursts into the network). The buffer which is an abstraction of the buckets hold tokens, in order for the host node to be able to transmit data, the host node first gets hold of the token, after which the token is destroyed. In order for the host to be able to send huge amounts of data, it remains idle and capture and save up tokens.

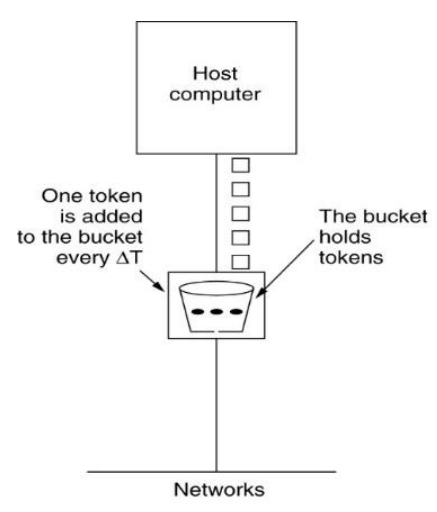

(a)

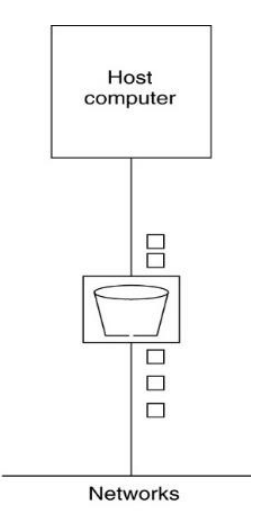

(b)

Figure 3: A representation of the Token bucket algorithm (Vidyasagar et al, 2013)

\section{Proposed Method of Implementation}

When considering the implementation of traffic shaping techniques into computer network systems, it is paramount to have an understanding of network system architecture. The algorithm is first embedded into the network architecture. Simulations should also be carried out first to see how it the algorithms functions in relation to network subsystems and network elements. From the simulation result obtain, we can then proceed to implementing it on a live scale network.

\section{Conclusion and Recommendations}

The congestion problem is an issue affecting a wide range of wireless applications. Most of the existing techniques are doing more harm than good. Having so high overhead and in turn adding more congestion to the system. Solutions with low overhead are needed. Traffic shaping algorithms have significantly low overhead and are very simple to implement, offering very little complexity. The implementation of traffic shaping algorithms using both the leaky bucket and the token bucket is an efficient measure of congestion control. Future research can be carried out in the development of more traffic shaping techniques.

\section{REFERENCES}

1 Alkharashi, A. (2016). Wireless \& Telecommunication. 2nd International Conference and Business Expo. Saudi Arabia: Open Access Jorunal.

2 Cardwell, N., Cheng, Y., Gunn, C. S., Yeganeh, S. H., \& Jacobson, V. (2016). Congestion-Based Congestion Control. acmqueue.

$3 \mathrm{Hu}, \mathrm{X} .$, \& Guo, W. (2016). Congestion Control in Wireless Software Defined Networks with Propagation Delay and External Interference: A Robust Control Approach. Hindawi Publishing Corporation.

4 Hussain, M. W., Jamwal, S., \& Zaman, M. (2015). Congestion Control Techniques in a Computer Network. International Journal of Computer Applications .

5 Jain, R. (1990). Congestion Control in Computer Networks: Issues and Trends. IEEE.

6 Jayakumari, B., \& Senthilkumar, J. (2015). Priority Based Congestion Control Dynamic Clustering Protocol in Mobile Wireless Sensor Networks. Hindawi Publishing Corporation.

7 Jiao, C., Gao, S., Yang, W., Xia, Y., \& Zhu, M. (2014). A Fast Heuristic Algorithm for Minimizing Congestion in the MPLS Networks. Scientific Research.

8 Majidi, A., \& Mirvaziri, H. (2014). A New Mechanism for Congestion Control in Wireless Multimedia Sensor Networks for Quality of 
Service and Network Life Time. American Journal of Computing Research Repository.

9 Meenatchi, I., \& Palanivel, K. (2014). Intrusion Detection System in MANETS: A Survey . International Journal of Recent Development in Engineering and Technology .

10 Miller, K., \& Hsiao, L. (2014). TCPTuner: Congestion Control Your Way. Stanford University Journal.

11 Mohamed, N., Sahib, S., Suryana, N., \& Hussin, B. (2016). Understanding Network Congestion Effects On Performance. Journal of Theoretical and Applied Information Technology.

12 Raja, L., \& Baboo, S. S. (2014). An Overview of MANET: Applications, Attacks and Challenges. International Journal of Computer Science and Mobile Computing .

13 Rysavy Research. (2014, March 09). LTE Congestion Management. Retrieved from Rysavy Research: . http://www.rysavy.com.

14 Sauter, M. (2011). From GSM to LTE: An Introduction To Mobile Networks and Mobile Broadband. West Sussex: Wiley.
15 Shahzad, F., Mushtaq, M. F., Ullah, S., Siddique, M. A., Khurram, S., \& Saher, N. (2015). Improving Queuing System Throughput Using Distributed Mean Value Analysis to Control Network Congestion. Scientific Research.

16 Shar, S. A., Nazir, B., \& Khan, I. A. (2016). Comgestiton Control Algorithms in Wireless Sensor Networks. Journal of King Saud University.

17 Sugeng, W., Istiyanto, J. E., Mustofa, K., \& Ashari, A. (2015). The Impact of QoS Changes towards Network Performance. International Journal of Computer Networks and Communications Security .

18 Ullah, S., Shahzad, F., Khurram, S., \& Anwer, W. (2014). Improving Network Efficiency by Selecting and Modifying Congestion Control Constraints. Scientific Research.

19 Vidyasagar, S., \& VidyaShankar, M. (2013). A study on 'Network Congestion Control Algorithms. Global Research Analysis.

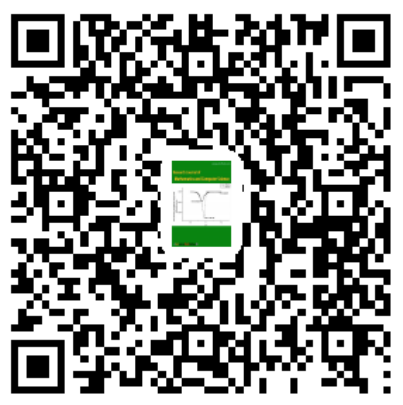

